\title{
Evidence for a positive relationship between perceived stress and belief in conspiracy theories
}

\author{
Matt N. Williams ${ }^{1 a}$, Rachel C. Anderson ${ }^{2}$, Nicholas D. Fox ${ }^{1}$, Chelsea M. Skinner ${ }^{1}$, \& \\ Brandon L. McMurtrie ${ }^{1}$
}

${ }^{1}$ School of Psychology, Massey University, New Zealand

${ }^{2}$ Te Ohu Rangahau Kai, AgResearch, New Zealand

aCorresponding author. Email: M.N.Williams@massey.ac.nz

\begin{abstract}
Perceived stress has previously been implicated in the belief of conspiracies, with some authors suggesting that stress can precipitate increased belief in conspiracy theories. This preregistered survey study aimed to replicate findings by Swami et al. (2016) showing a positive correlation between belief in conspiracy theories and perceived stress. 372 Australian and New Zealand residents participated. Beliefs in conspiracy theories were measured using 11 items drawn mainly from existing scales ( $\alpha$ $=.87$ ). Perceived stress was measured using the 10-item Perceived Stress Scale (PSS; $\alpha$ $=.91)$. The average level of endorsement of conspiracy theories was relatively low ( $M=$ 1.89 on a Likert scale from 1 to $5 ; S D=0.72$ ). Supporting our hypothesis, we found a significant and positive correlation between perceived stress and belief in conspiracy theories, $r(370)=.20, p=.001,95 \%$ CI [.096, .292]. Future work could investigate the causal mechanism producing this association.
\end{abstract}




\section{Introduction}

A conspiracy theory claims that "multiple actors are intentionally plotting to accomplish malevolent goals" (Swami et al., 2016, p. 76). Conspiracies do happen, and in some cases the best explanation for a real-life event is a conspiracy theory (see Dentith, 2018). However, beliefs in unwarranted conspiracy theories (theories which are not supported by empirical evidence) are relatively common in the general population (see Douglas et al., 2017; Marques et al., 2021). Exposure to such conspiracy theories can cause harm (Douglas, 2021). Experimental evidence suggests that exposure to conspiracy theories can reduce intentions to reduce one's carbon footprint (Jolley \& Douglas, 2014b), decrease health-seeking intentions (Natoli \& Marques, 2020), and reduce vaccination intentions (Jolley \& Douglas, 2014a).

One feature of belief in conspiracy theories of particular interest to psychologists is a potential relationship between a tendency to believe in conspiracy theories and psychological stress - i.e., the perception that "environmental demands exceed [one's] adaptive capacity" (Cohen et al., 2007, p. 1685). Based on a review of historical incidents such as the great fire of Rome and the rise of the Nazi party in Germany, van Prooijen and Douglas (2017) suggest that during times of crisis, the tendency to believe in conspiracy theories increases. They theorise that belief in conspiracy theories can serve as a coping mechanism to deal with the distressing feelings which come from difficult and uncertain circumstances. In such circumstances, conspiracy theories can help us cope by facilitating sense-making - i.e., by providing explanations for stressful circumstances. Unfortunately, the belief that such conspiracies may be true could be a sense of threat in itself, causing stress rather than just alleviating it (van Prooijen, 2019).

A small quantity of empirical research has sought to determine whether there is indeed a positive relationship between beliefs in conspiracy theories and perceived stress (i.e., individuals' subjective appraisal of their current levels of stress)ํ. A notable study in this area was conducted by Swami et al. (2016). Based on a survey of 420 US

${ }^{1}$ Given that the definition of psychological stress we have provided above (from Cohen et al., 2007) refers to perceptions of stress, we regard "psychological stress" and "perceived stress" as synonymous in this context. 
adults, Swami et al. found that belief in conspiracy theories was positively correlated with perceived stress ( $r=.15$, when controlling for age and subjective social status).

Although Swami et al.'s study was correlational, they (like van Prooijen \& Douglas, 2017) tentatively inferred that stress may cause increased belief in conspiracy theories: "this view suggests that stressful events give rise to sensemaking processes that favour conspiracy theories.... stressful intra-individual life events may sometimes lead to a tendency to adopt a conspiracist mind-set" (p. 74). Their application of controls for age and subjective social status seemingly represents an attempt to control for two plausible confounds.

Despite Swami et al.'s findings, subsequent studies have implied the existence of ambiguity about whether there is a relationship between perceived stress and belief in conspiracy theories. Survey studies by Georgiou et al. (2020) and Ferreira et al. (2020) both found no significant bivariate relationship between perceived stress and belief in conspiracy theories relating to COVID-19. This said, Braud et al. (2021) aimed to replicate the findings of Georgiou et al. (2020), and uncovered several errors in their analysis approach (including inconsistent numeric figures). Further preregistered analyses by Braud et al. using a small sample of 237 participants found perceived stress to be positively related to belief in COVID-19 conspiracy beliefs (when controlling for assessment of government response and education). Similarly, in a sample from Greece and Cyprus ( $N=1001)$, Constantinou et al. (2021) found a significant but small ( $r=.13$ ) positive correlation between distress about COVID-19 and belief in conspiracy theories. An Italian study (Simione et al., 2021) likewise reported a significant correlation ( $r=$ $.20, p<.01$ ) between perceived stress and belief in conspiracy theories.

Considering this literature in combination, there remains some ambiguity about whether perceived stress and beliefs in conspiracies are correlated at a bivariate level (and, if so, whether this correlation is of a meaningful size). Establishing such a correlation is an important step prior to subsequent studies that may test hypotheses that explain the mechanism of such a relationship. It is furthermore important that this relationship is established in well-powered preregistered studies, which can provide readers with greater confidence that results have not been selectively reported to suit a desired conclusion (see Nosek et al., 2018). In this study, we therefore aimed to test the 
preregistered hypothesis belief in conspiracies is positively correlated with perceived stress (H1).

In addition to this hypothesis, we preregistered three supplementary research questions:

- RQ1: To what extent do Australians and New Zealanders believe in a range of well-known conspiracy theories?

- RQ2: What is the internal consistency reliability of responses to the conspiracy beliefs scale used in this study?

- RQ3: What is the internal consistency reliability of responses to the perceived stress scale?

\section{Method}

\section{Participants and Procedure}

This study used a cross-sectional survey design. Participants were recruited from Prolific (prolific.co), a participant-recruitment website, with the survey completed in Qualtrics. Participants were paid £o.6o each. Pre-screening inclusion criteria were specified in Prolific such that only participants currently living in Australia or New Zealand and having at least a 95\% approval rate on prior tasks were eligible ${ }^{2}$. The inclusion criterion of living in Australia or New Zealand was specified in order to ensure that the study materials were culturally and linguistically suited to the participants (the researchers are located in Australasia), and to allow data collection from outside the traditionally oversampled regions of Europe and North America.

A target sample size was determined via a power analysis for a correlation (testing H1). This power analysis suggested that a sample size of 346 would be required to obtain $80 \%$ power to detect a true correlation of .15 in a two-tailed test. $r=.15$ is the size of the partial correlation between perceived stress and belief in conspiracies in Swami et al. (2016). To ensure that this sample size was met, a target sample size of 387

\footnotetext{
${ }^{2}$ Prolific operationalises the approval rate as the upper limit of the 95\% confidence interval for
} the participant's approval rate based on prior tasks. 
was specified in Prolific. This allowed for the possibility that a small quantity of data (an estimated maximum of 10\%) would need to be excluded based on our exclusion criteria.

A total of 388 participants' responses were initially collected. These were then subject to the following preregistered exclusion criteria:

1. Having a Qualtrics "status" indicating a test, preview, or suspected duplicate response

2. Not being aged 18 or over

3. Not currently residing in Australia or New Zealand

4. Not responding "Somewhat agree" to an attention check item directing this response

5. Not reaching the end of the survey

6. Not consenting to participate.

The exclusion criteria resulted in the exclusion of 16 participants, and a final sample size of 372 . The majority of the participants, $80.6 \%$, were currently living in Australia, with the remaining 19.4\% living in New Zealand. 55.4\% identified as male, 43.0\% as female and $1.6 \%$ as nonbinary/gender diverse. Participants' ages ranged from 18 to 82 years, with a median of 31 years. There was no missing data in the final sample.

This study included a follow-up component where a small subset of the sample was invited to complete the conspiracy items again a month later, serving as a pilot for a longitudinal study. For the sake of brevity, we do not report data from the follow-up component here.

\section{Measures}

\section{Belief in Conspiracy Theories}

Participants were asked to rate the extent to which they agreed with 11 statements describing conspiracy theories, with responses on a five-point Likert-type response scale (see Figure 1 for the items). The items were selected due to their contemporary relevance to Australians and New Zealanders and were drawn from previous studies on beliefs in conspiracies (Lewandowsky et al., 2013; Marques et al., 2021; Swami et al., 
2017), as well as one new item written for this study. A belief in conspiracy theories score was calculated by taking the mean of each participant's responses (possible range $1-5)$.

\section{Perceived Stress}

Replicating the approach of Swami et al. (2016), we measured perceived stress using the Perceived Stress Scale (PSS; Cohen et al., 1983), which contains 10 items. An example item is "In the last month, how often have you felt nervous and stressed?" Responses were collected on a 5 -point rating scale ( $0=$ never, $4=$ very often). The PSS is a widely used scale and has acceptable psychometric properties (Lee, 2012). A perceived stress score was calculated by taking the mean of each participant's responses (possible range 0-4).

\section{Ethics}

Our study protocol was peer-reviewed and determined to be low risk according to the criteria of our institution. A low-risk notification (similar to "exempt review" in North America) was lodged with the institutional ethics committee.

\section{Preregistration and data availability statement}

A detailed preregistration, de-identified data and an analysis script are available at https://osf.io/z8exd/

\section{Results}

\section{Descriptive Statistics}

Descriptive statistics and estimates of internal consistency reliability (Cronbach's alpha) for each of the two measures are provided in Table 1. In general, participants tended to disagree with the conspiracy theory items (median $=1.73$ ).

Table 1

Descriptive Statistics 


\begin{tabular}{lrrrrrr}
\hline Variable & $\begin{array}{r}\text { Possible } \\
\text { range }\end{array}$ & M & Median & SD & Skew & Alpha \\
\hline $\begin{array}{l}\text { Belief in conspiracy } \\
\text { theories }\end{array}$ & $1-5$ & 1.89 & 1.73 & 0.72 & 0.86 & 0.87 \\
\begin{tabular}{l} 
Perceived stress \\
\hline
\end{tabular} & $0-4$ & 1.73 & 1.70 & 0.76 & 0.52 & 0.91 \\
\hline
\end{tabular}

The distributions of responses to individual items are displayed in Figure 1. Some theories were much more popular than others, but no conspiracy theory was endorsed by more than a quarter of the sample.

\section{Confirmatory Analysis}

As per the preregistration, hypothesis 1 was tested using a Pearson's productmoment correlation between perceived stress score and belief in conspiracies score. This correlation was positive and statistically significant, $r(370)=.20, p<.001,95 \%$ CI [.10, .29], supporting the hypothesis. 


\section{Figure 1}

\section{Likert Bar Plot for Participants' Responses to Conspiracy Theory Items}

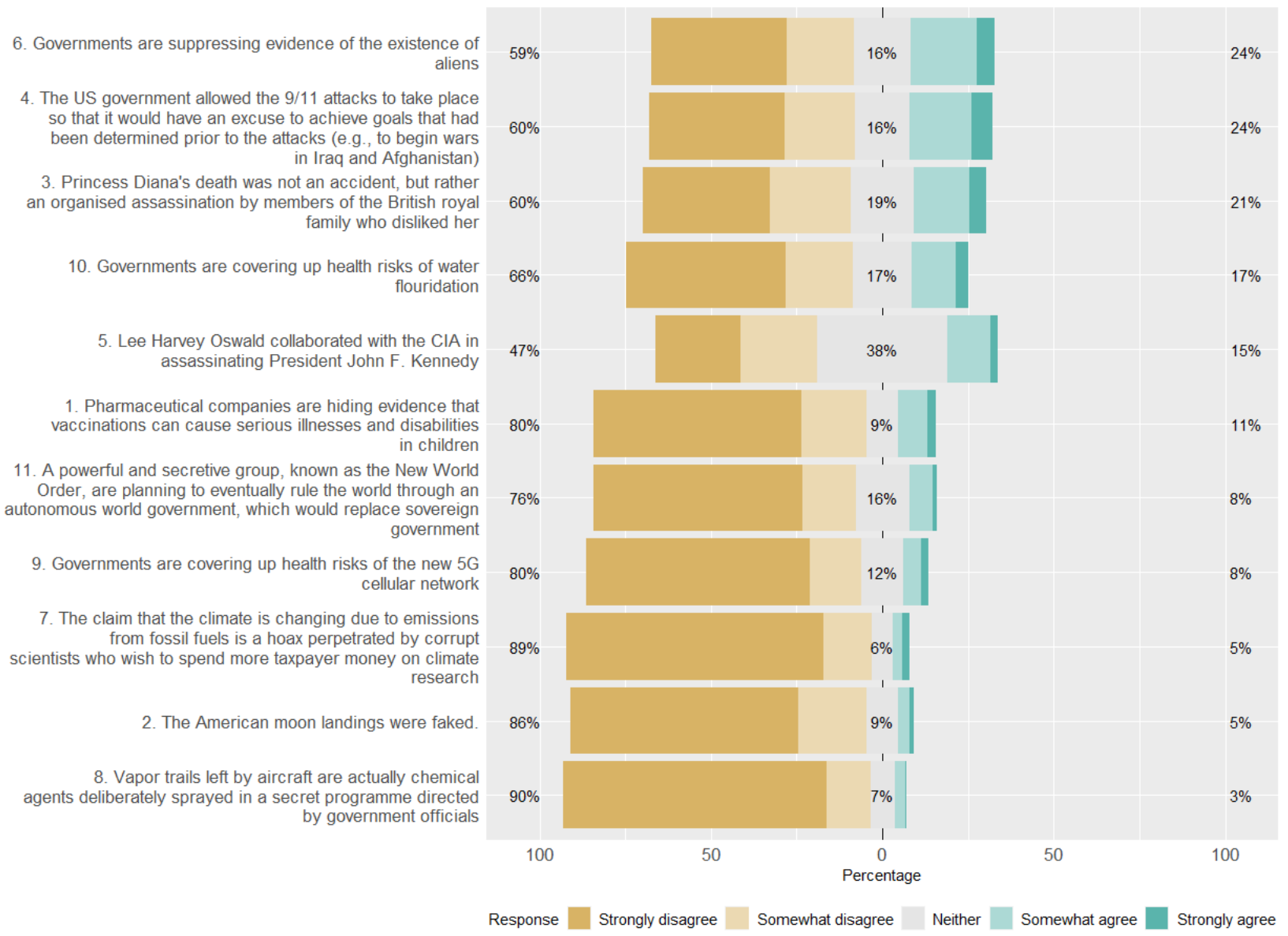

Notes. The percentages on the left indicate the percentage disagreeing (strongly or somewhat). The percentages on the right indicate the percentage agreeing (somewhat or strongly). Items are displayed in descending order of agreement. Items 1, 2, 5, 6, 8, and 9 are from Marques et al. (2021), items 3, 4, and 11 are adapted from Swami et al. (2017), and item 7 is from Lewandowsky et al. (2013). Item 10 was created for this study.

\section{Robustness Analyses}

Several non-preregistered analyses were conducted to check the robustness of our findings to alternative specifications. First, the confidence interval for the Pearson's correlation was estimated via percentile bootstrapping. This loosened the assumption of bivariate normality, and resulted in a nearly identical 95\% confidence interval, [.10, .29]. Second, the correlation between belief in conspiracy theories and perceived stress was estimated using Spearman's rho. This loosened the assumption of linearity. This resulted in a nearly identical correlation, $r=.20, p<.001$. Finally, a structural equation 
model was estimated where all PSS items loaded on a "perceived stress" latent variable, all conspiracy theory items loaded on a "belief in conspiracy theories" latent variable, and the two latent variables were correlated with one another. This model accounted for the effects of measurement error. The correlation between stress and belief in conspiracy theories remained nearly identical to that in the original analysis, $r=.21, p=$ .001.

\section{Discussion}

In agreement with the hypothesis, the results showed a positive and statistically significant correlation between perceived stress and belief in conspiracies. The size of this zero-order correlation was similar to that of the partial correlation reported by Swami et al. (2016) for US participants (.20 versus .15). It is likewise very similar to the correlation of .20 found in Simione et al. (2021). Considering our findings along with the existing literature it seems reasonable to infer that there is now robust evidence of a positive relationship between perceived stress and beliefs in conspiracies - but that this correlation is relatively small.

Our study had several strengths. It was preregistered, used a relatively large sample, and our robustness analyses suggest our findings are highly robust to alternative specification decisions. Nevertheless, it also had limitations. First, our sample was a non-representative convenience sample. Compared to the overall Australian and New Zealand populations (Australian Bureau of Statistics, 2017; Stats NZ, n.d.), the participants in this study were slightly younger and a larger percentage were male. We did not collect information about other demographic factors such as ethnicity, socio-economic status and education level. Second, our measure of belief in conspiracy theories was not pre-validated - although it did display satisfactory internal consistency reliability (as did the PSS). Third, our study sought only to establish a correlation between perceived stress and belief in conspiracy theories, and cannot determine the causal mechanism producing this correlation. Future studies could use experimental, longitudinal or experience-sampling approaches to test hypotheses regarding this mechanism. 


\section{References}

Australian Bureau of Statistics. (2017). 2016 census quickstats: Australia. https://quickstats.censusdata.abs.gov.au/census_services/getproduct/census/2 016/quickstat/o36?opendocument

Braud, M., Gaboriaud, A., Ferry, T., Mardi, W. E., Silva, L. D., Lemouzy, M., Guttierrez, J., Petit, S., Szabelska, A., \& IJzerman, H. (2021). COVID-19-related conspiracy beliefs and their relationship with perceived stress and pre-existing conspiracy beliefs in a Prolific Academic sample: A replication and extension of Georgiou et al. (2020). PsyArXiv. https://doi.org/10.31234/osf.io/t62s7

Cohen, S., Janicki-Deverts, D., \& Miller, G. E. (2007). Psychological stress and disease. JAMA, 298(14), 1685-1687. https://doi.org/10.1001/jama.298.14.1685

Cohen, S., Kamarck, T., \& Mermelstein, R. (1983). A global measure of perceived stress. Journal of Health and Social Behavior, 24(4), 385-396. https://doi.org/10.2307/2136404

Constantinou, M., Gloster, A. T., \& Karekla, M. (2021). I won't comply because it is a hoax: Conspiracy beliefs, lockdown compliance, and the importance of psychological flexibility. Journal of Contextual Behavioral Science, 20, 46-51. https://doi.org/10.1016/j.jcbs.2021.03.001

Dentith, M. R. X. (Ed.). (2018). Taking conspiracy theories seriously. Rowman \& Littlefield.

Douglas, K. M. (2021). Are conspiracy theories harmless? The Spanish Journal of Psychology, 24(e13). https://doi.org/10.1017/SJP.2021.10

Douglas, K. M., Sutton, R. M., \& Cichocka, A. (2017). The psychology of conspiracy theories. Current Directions in Psychological Science, 26(6), 538-542. 
https://doi.org/10.1177/0963721417718261

Ferreira, S. P. R., Campos, C., Marinho, B. M., Rocha, S., Fonseca-Pedrero, E., \& Rocha, N. (2020). What drives beliefs in COVID-19 conspiracy theories? The role of psychotic-like experiences and confinement-related factors. PsyArXiv. https://doi.org/10.31234/osf.io/2mjye

Georgiou, N., Delfabbro, P., \& Balzan, R. (2020). COVID-19-related conspiracy beliefs and their relationship with perceived stress and pre-existing conspiracy beliefs. Personality and Individual Differences, 166, 110201.

https://doi.org/10.1016/j.paid.2020.110201

Jolley, D., \& Douglas, K. M. (2014a). The effects of anti-vaccine conspiracy theories on vaccination intentions. PloS One, 9(2), e89177. https://doi.org/10.1371/journal.pone.0o89177

Jolley, D., \& Douglas, K. M. (2014b). The social consequences of conspiracism: Exposure to conspiracy theories decreases intentions to engage in politics and to reduce one's carbon footprint. British Journal of Psychology, 105(1), 35-56. https://doi.org/10.1111/bjop.12018

Lee, E.-H. (2012). Review of the psychometric evidence of the Perceived Stress Scale. Asian Nursing Research, 6(4), 121-127. https://doi.org/10.1016/j.anr.2012.08.004

Lewandowsky, S., Gignac, G. E., \& Oberauer, K. (2013). The role of conspiracist ideation and worldviews in predicting rejection of science. PLOS ONE, 8(10), e75637. https://doi.org/10.1371/journal.pone.0075637

Marques, M. D., Ling, M., Williams, M. N., Kerr, J. R., \& McLennan, J. (2021). Australasian public awareness and belief in conspiracy theories: Motivational 
correlates. Political Psychology. https://doi.org/10.1111/pops.12746

Natoli, E. E., \& Marques, M. D. (2020). The antidepressant hoax: Conspiracy theories decrease health-seeking intentions. British Journal of Social Psychology, Advance online article. https://doi.org/10.1111/bjso.12426

Nosek, B. A., Ebersole, C. R., DeHaven, A. C., \& Mellor, D. T. (2018). The preregistration revolution. Proceedings of the National Academy of Sciences, 115(11), 26002606. https://doi.org/10.1073/pnas.1708274114

Simione, L., Vagni, M., Gnagnarella, C., Bersani, G., \& Pajardi, D. (2021). Mistrust and beliefs in conspiracy theories differently mediate the effects of psychological factors on propensity for COVID-19 vaccine. Frontiers in Psychology, o. https://doi.org/10.3389/fpsyg.2021.683684

Stats NZ. (n.d.). Place summaries | New Zealand. Retrieved July 13, 2021, from https://www.stats.govt.nz/tools/2018-census-place-summaries/new-zealand

Swami, V., Barron, D., Weis, L., Voracek, M., Stieger, S., \& Furnham, A. (2017). An examination of the factorial and convergent validity of four measures of conspiracist ideation, with recommendations for researchers. PLOS ONE, 12(2), e0172617. https://doi.org/10.1371/journal.pone.0172617

Swami, V., Furnham, A., Smyth, N., Weis, L., Lay, A., \& Clow, A. (2016). Putting the stress on conspiracy theories: Examining associations between psychological stress, anxiety, and belief in conspiracy theories. Personality and Individual Differences, 99, 72-76. https://doi.org/10.1016/j.paid.2016.04.084 van Prooijen, J.-W. (2019). An existential threat model of conspiracy theories. European Psychologist. van Prooijen, J.-W., \& Douglas, K. M. (2017). Conspiracy theories as part of history: The 
role of societal crisis situations. Memory Studies, 1O(3), 323-333.

https://doi.org/10.1177/1750698017701615 